\title{
Controlled growth mechanism of poly (3-hexylthiophene) nanowires
}

\author{
D Kiymaz ${ }^{1}$, M Yagmurcukardes ${ }^{2}$, A Tomak $^{3}$, H Sahin ${ }^{4}$, R T Senger ${ }^{2}$, \\ F M Peeters ${ }^{4}$, H M Zareie ${ }^{3,5}$ and C Zafer ${ }^{1}$ \\ ${ }^{1}$ Solar Energy Institute, Ege University, 35100 Izmir, Turkey \\ ${ }^{2}$ Department of Physics, Izmir Institute of Technology, 35430 Urla, Izmir, Turkey \\ ${ }^{3}$ Department of Materials Science and Engineering, Izmir Institute of Technology, 35430 Urla, Izmir, \\ Turkey \\ ${ }^{4}$ Department of Physics, University of Antwerp, Groenenborgerlaan 171, B-2020 Antwerp, Belgium \\ ${ }^{5}$ Microstructural Analysis Unit, School of Physics and Advanced Materials, University of Technology, \\ Sydney, Ultimo NSW 2007, Australia \\ E-mail: ceylan.zafer@ege.edu.tr
}

Received 5 May 2016, revised 1 September 2016

Accepted for publication 12 September 2016

Published 11 October 2016

\begin{abstract}
Synthesis of 1D-polymer nanowires by a self-assembly method using marginal solvents is an attractive technique. While the formation mechanism is poorly understood, this method is essential in order to control the growth of nanowires. Here we visualized the time-dependent assembly of poly (3-hexyl-thiophene-2,5-diyl) (P3HT) nanowires by atomic force microscopy and scanning tunneling microscopy. The assembly of P3HT nanowires was carried out at room temperature by mixing cyclohexanone $(\mathrm{CHN})$, as a poor solvent, with polymer solution in 1,2dichlorobenzene (DCB). Both $\pi-\pi$ stacking and planarization, obtained at the mix volume ratio of P3HT (in DCB):CHN (10:7), were considered during the investigation. We find that the length of nanowires was determined by the ordering of polymers in the polymer repetition direction. Additionally, our density functional theory calculations revealed that the presence of DCB and CHN molecules that stabilize the structural distortions due to tail group of polymers was essential for the core-wire formation.
\end{abstract}

Keywords: self assembly, nanowire, poly(3-hexyl-thiophene), $\pi-\pi$ stacking

(Some figures may appear in colour only in the online journal)

\section{Introduction}

Through the past decade, conjugated conducting polymers have been attractive materials for organic electronic devices such as solar cells [1], light emitting diodes, and field effect transistors (FETs) [2] because of their solution processability, excellent film forming properties, good charge transport properties, etc. Polythiophenes, especially poly (3-hexylthiophene-2, 5-diyl) (P3HT), have attracted a lot of interest due to their relatively good stability and higher mobility. P3HT has become the benchmark p-type semiconductor in organic photovoltaics due to these advantages. However, limitations such as short exciton diffusion length and narrow optical absorption coverage, affect the energy conversion efficiencies of P3HT dramatically. Thus the film thickness of the donor material should be optimized in order to have enough light absorption and efficient charge collection upon effective exciton dissociation. This requirement leads researchers to focus on 1D ordered organic nanostructures, such as nano-rods [3], nanofibers and nanowires [4], where the aim is for the charges to be transported faster through the nanowires/nanofibres with lower recombination rates [5].

Molecular self-assembly is one of the most widely used methods for nanoscale morphology design due to controllable structure architecture and easy production. The mechanism of solution phase self-assembly is explained in terms of polymer interactions [4, 6-8]. When poor solvent is added to polymer solution, P3HT polymers tend to minimize the unfavorable contacts between backbone and hydrocarbon solvent due to the solvophobic interactions which results in polymer chains getting closer and increasing the chain-chain interaction [9]. 
Therefore, random $\pi-\pi$ stacking between polymer backbones and between the alkyl side chains, by van der Waals interactions, are initiated via diffusive motion [7]. However 'critical mass' limits the nanowire formation. Johns et al determined that, to start the formation of stable nanowire, at least about 60 monomers should exist within the interacting chain segment of the polymer aggregates. Interaction of polymer chains consisting of less than 60 monomers, causes the breaking of the bonds between the polymers due to thermal motion, and polymers become independent from each other [8]. If the required critical mass is provided, then aggregation begins and polymers bind along the $\pi-\pi$ stacking direction in microseconds forming stable-core wires [6].

Kiriy et al claim that at high poly alkyl thiophene (PAT) concentrations in poor solvents, PAT molecules aggregate in face to face configuration to form rod like nanostructures in order to minimize the interaction with poor solvent and aromatic thiophene ring. Furthermore at low PAT concentrations, PAT molecules adopt a helical conformation [9].

To date, optical properties and increment in charge mobility, as well as limitation in exciton diffusion length, of poly (3-alkylthiophenes) (P3AT) [10] and P3HT nanowires [11] have been investigated experimentally.

Qiu et al [15] have reported an assembly of polymer backbones parallel to the wire axis under electric field during annealing at $200{ }^{\circ} \mathrm{C}$. The polymers were attached end to end due to their dipole attractions at the polymer ends under electric field to form high crystallinity. The electrical conductivity of the electric field annealed P3HT films is higher more than one order of magnitude compared to thermal annealed or non-annealed films.

Although these studies were focused on the clarification of properties and dynamics of nanowires [17], there are still unclarified aspects of the nanowire creation mechanism for both situation of crystallization. In this study we demonstrate the self-assembly behavior of P3HT polymers whose backbones are parallel to the nanowire axis, by utilizing the poor solvent approach. Nanowires are prepared by adding cyclohexanone $(\mathrm{CHN})$, which is a poor solvent, on P3HT solution in 1,2-dichlorobenzene (DCB), and the resultant self-assembly formations were characterized by atomic force microscopy (AFM) and scanning tunneling microscopy (STM) in different incubation times. Growth of the P3HT nanowires was observed to be affected by the ratio of the poor and good solvent and time. Here we focused on the packing behavior of P3HT polymers and found that the polymers are ordered parallel with the wire axis, while wire diameter is determined by chain-chain interaction. For a deeper understanding of the solvent assisted formation of P3HT nanowires at atomic level, we also performed $a b$ initio theory calculations. We found that, for the stabilization of lamellar structure of P3HT nanowires, the presence of DCB and CHN solvent molecules plays an important role. In the final part, we investigate the effect of interface area between poor solvent and polymer solution on nanowire diameter.

\section{Experimental methods}

\subsection{Materials}

The regioregular poly (3-hexyl-thiophene-2,5-diyl) (rr-P3HT) was purchased from American Dye Source, Inc. and used without further purification. Cyclohexanone ( $\mathrm{CHN}$ ) was purchased from AKS, Atabay Pharmaceutical Products Inc., Turkey and used as a poor solvent while 1,2-dichlorobenzene (DCB) was purchased from Sigma-Aldrich, USA and used as a main solvent for rr-P3HT polymer.

\subsection{Synthesis of P3HT-nanowires}

$20 \mathrm{mg} \mathrm{ml}^{-1}(2 \% \mathrm{w} / \mathrm{v})$ of P3HT was dissolved in DCB and prepared solution bubbled with inert Ar gas to remove oxygen. The polymer solution was stirred at $40^{\circ} \mathrm{C}$ for $3 \mathrm{~h}$ to dissolve the polymers completely and cooled down to room temperature. Upon adding $70 \mu \mathrm{l}$ non-solvent pure $\mathrm{CHN}$ on $100 \mu \mathrm{l}$ P3HT solution, the red-orange color of P3HT solution was slowly changed to brown and then to dark purple due to increase of the interaction between P3HT chains leading the generation of P3HT aggregates. After the addition of $\mathrm{CHN}$ to the polymer solution, $10 \mu \mathrm{l}$ final solution was spin coated on mica surface at $4000 \mathrm{rpm}$ for $60 \mathrm{~s}$ at specific time intervals; $1 \mathrm{~s}, 1,2,4,8,15$ and 30 min Afterwards, the substrates were dried at $60{ }^{\circ} \mathrm{C}$ for $15 \mathrm{~min}$ in a vacuum. The morphology of the prepared films was investigated by scanning probe microscopy (AFM/STM).

In the second part of the study, two vials with different diameters were used for understanding the generation mechanism depended on the interaction area between $\mathrm{CHN}$ and P3HT dissolved in 1, 2 DCB $\left(20 \mathrm{mg} \mathrm{ml}^{-1}\right) .100 \mu \mathrm{l}$ of P3HT solutions were put in vials with 11 and $15 \mathrm{~mm}$ diameter in order to obtain different areas of P3HT/CHN interface. Then $70 \mu \mathrm{l}$ of $\mathrm{CHN}$ was added to the both vials and the solutions were left overnight. Finally, nanowire-containing solutions were spin coated on mica substrates at $4000 \mathrm{rpm}$ for AFM investigations.

\subsection{UV-vis absorption}

Optical absorbance of P3HT in DCB: CHN mixture was recorded with $\mathrm{UV} / \mathrm{Vis}$ spectrophotometer (Analytic Jena S600 UV/Vis) by sandwiching the mixture solution between glass slides.

\subsection{Atomic force microscopy}

A Nanosurf FlexAFM system and Ambious Technology Q-Scope 250 instrument operating in tapping mode under ambient conditions was used to obtain topographic data. All imaging operations were conducted with $512 \times 512$ data acquisitions at a various scan speed. Oxide-sharpened silicon nitride tips with integrated cantilever with a nominal spring constant of $48 \mathrm{~N} \mathrm{~m}^{-1}$ were used. These tips had resonance frequencies between 146 and $236 \mathrm{kHz}$ and an effective radius of curvature at the tip of less than $7 \mathrm{~nm}$. AFM images were manipulated by using Scanning Probe Image Processor 
(SPIP) software. For morphology studies, mica substrates (Mica-Grade V-4 SPI, Catalogue number 1869-CA and Lot number 1170203 ) were used. They were cleaned by ultrasonification for $15 \mathrm{~min}$ in isopropyl alcohol and distilled water respectively, and dried by high purity nitrogen blow.

\subsection{Scanning tunneling microscopy}

STM images were acquired using a Nanosurf EasyScan system under ambient conditions. STM piezoelectric scanners were calibrated laterally with graphite and $\mathrm{Au}$ (111), and vertically by using the height of the Au (111) steps $(2.2 \AA)$. The STM tip was prepared from $\mathrm{Pt} / \mathrm{Ir}$ (90\%/10\%) $0.25-0.2 \mathrm{~mm}$ wire cut under ambient conditions. All images were acquired in a constant-current mode. Typical imaging conditions were bias voltage of $1,7-2,5 \mathrm{~V}$ and a tunneling current of 100-800 pA. Images were manipulated with Scanning Probe Image Processor (SPIP) software.

\section{6. $X$-ray diffraction $(X R D)$ analysis}

Investigation of the crystal structure of P3HT nanowires was performed by a high resolution Rigaku Ultima IV x-ray Difractometer. The wavelength of $\mathrm{CuK}-\alpha$ x-rays was $1.542 \AA$. The energy of focused beam was $0.8 \mathrm{keV}$ and the detector was scintillation counter. Measurements were taken in out of plane mode by using cross-beam optics.

\section{Computational methodology}

Structural optimizations and determination of electronic ground states were carried out by performing first principles calculations within density functional theory [18] using the Spanish Initiative for Electronic Simulations with Thousands of Atoms package $[19,20]$. All the calculations were performed using pseudopotentials with local basis functions and the local density approximation [21, 22] for the exchangecorrelation potential [23]. The interactions between electrons and core ions were simulated with separable Troullier-Martins [24] norm-conserving pseudopotentials. For the geometry relaxations of $1 \mathrm{D}$ and 2D P3HT layers, a rectangular primitive unit cell was considered (see figures 3(B) and (C)). The geometry optimization of P3HT monomer, including 25 atoms, was perfomed with inserting vacuum in all directions. Then, two P3HT monomers (50 atoms), bonded from thiophene sites (see figure $3(\mathrm{~A})$ ), were geometrically relaxed in a vacuum. For the $1 \mathrm{D}$ chain of the monomers (see figure 3(B)) optimization was achieved by considering again 50 atoms in a primitive unit cell which is periodic along the $a$-vector. The 2D periodic monolayer P3HT structure was optimized by considering a rectangular unit cell containing 100 atoms with the vacuum present only in the vertical direction. Finally, bulk structure of P3HT (see figure 8) containing DCB and CHN molecules was optimized in the same primitive unit cell (with 79 atoms). The structural optimization of DCB and CHN molecules was investigated in the same primitive unit cell with inserting vacuum in three directions. The minimum

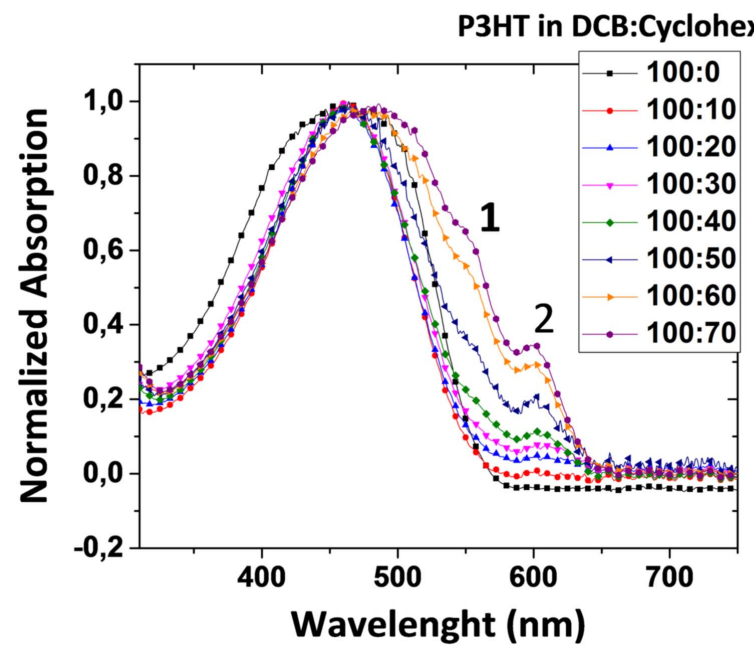

Figure 1. Normalized UV-vis absorption spectrum of P3HT (in DCB): CHN (10:X, X:1-7 in volume ratio) mixtures which were sandwiched between glass slides.

energy was calculated for various lattice constants and the pressure in all directions decreased to below 1 kbar. The optimization of atomic positions was performed by minimizing the total energy and the forces on the atoms hence the energy difference between sequential steps was taken $10^{-4} \mathrm{eV}$ as a convergence criterion in the structural relaxation, and the convergence for the Hellmann-Feynman forces on each atom was taken to be $0.04 \mathrm{eV} \AA^{-1}$.

Electronic optimization of $\mathrm{P} 3 \mathrm{HT}$ nanowires was performed by determining the electrostatic potentials on a real space grid with a mesh cut off energy of $500 \mathrm{Ry}$. The Brillouin zone was sampled with $5 \times 1 \times 1$ points within the Monkhorst-Pack $k$-point sampling scheme for 1D layer and $2 \times 2 \times 1$ for 2D P3HT. For the determination of density of states smearing parameter of 0.01 was used to obtain more accurate band gap energy for periodic structures of P3HT and accurate HOMO-LUMO gap for the DCB and CHN molecules. All the calculations were performed within the spin degenerate mode since the investigated structures are nonmagnetic.

\section{Results and discussion}

\subsection{UV-vis absorption spectroscopy and XRD analysis}

Figure 1 represents the normalized absorption spectra of P3HT solutions. In order to obtain a poor solvent effect, different amounts of $\mathrm{CHN}$ were added on individual P3HT solutions in DCB and the mixtures were kept for $24 \mathrm{~h}$. Typically, single absorption band around $\lambda=463 \mathrm{~nm}$ was observed for the $\pi-\pi^{*}$ transition of fully dissolved P3HT chains $[13,14]$ After addition of the CHN nonsolvent, additional absorption bands at $\lambda=555 \mathrm{~nm}$ (pointed with (1) and $602 \mathrm{~nm}$ (pointed with (2)), were observed. These absorption bands are related to the formation of crystalline like nanofibers. According to UV-vis absorption spectra analysis, peaks at $602 \mathrm{~nm}$ which indicate the interchain vibronic mode [13] 
A

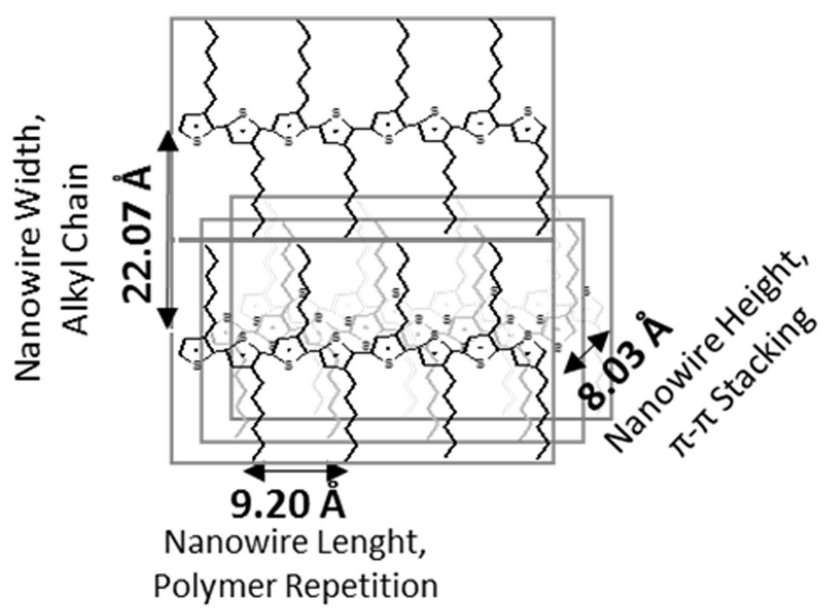

B

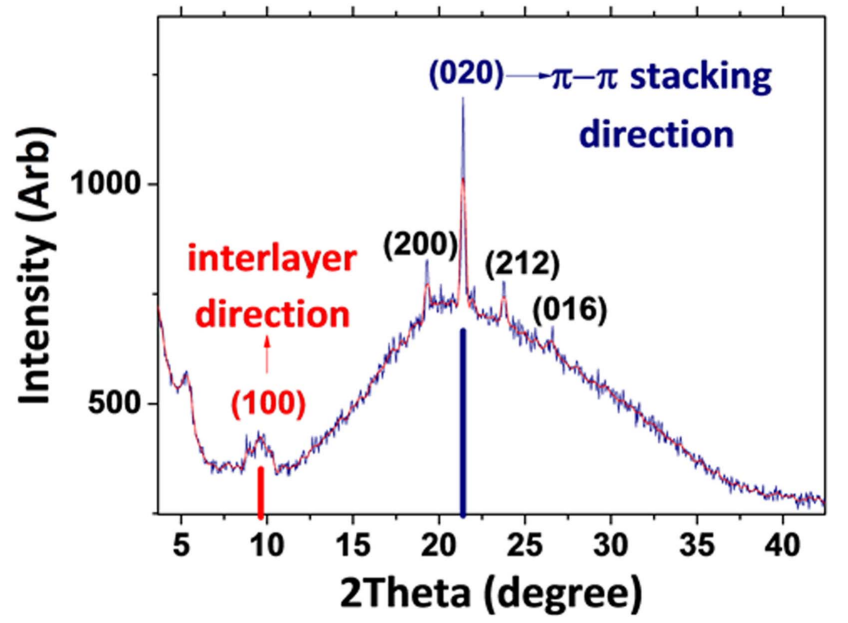

Figure 2. (A) Schematic illustration of P3HT nanowires and (B) in-plane XRD measurement graph of P3HT nanowire.

due to $\pi-\pi$ stacking, were observed at 100:40 (P3HT in DCB: $\mathrm{CHN}$ ) solvent mixture where ordered structure was initiated by self-organization. Besides that, the shoulders at $555 \mathrm{~nm}$ were attributed to intrachain vibronic mode [14] These shoulders were slightly seen at (100:60) and (100:70) mixtures as a result of planarization.

In order to identify the crystallographic structure of P3HT nanowires, in-plane grazing incident XRD analysis was carried out. According to the XRD analysis (figure 2(B)), the peak at $9.65^{\circ}$ corresponds to interlayer distance of the (100) diffraction whereas the strong peak at $23.8^{\circ}$ is attributed to $\pi-$ $\pi$ stacking direction corresponding to the (020) diffraction. With respect to XRD pattern, the distance between alkyl side chains (d100) is $22.07 \AA$ and calculated stack distance (d020) is $8.03 \AA$. The distance of the thiophene reputation is $9.20 \AA$ which is determined by the polymer backbone chain length.

\subsection{Theoretical calculations}

The nanowire growth occurs in microsecond range upon addition of poor solvent into P3HT solution [12], and the simulations of CHN effect assist to clarify the growth mechanism of core-nanowires. In line with this purpose, DFT calculations were performed in three main simulations. Firstly the structural properties of bulk P3HT were investigated by different stacking orientations. Thereafter we have focused on the interaction of DCB molecule with the stacked P3HT layers which constitute the bulk P3HT in atomic scale. Finally, effect of CHN molecule on the P3HT-DCB mixed structure was investigated in order to understand the formation of P3HT nanowire.

Results of the calculations demonstrate that firstly two P3HT molecules bonded from the thiophene sites, see figure 3(A), were optimized. In this case vacuum spacing was inserted in three directions so that the P3HT molecules are isolated. The atomic bond lengths were calculated to be $1.51 \AA$ for $\mathrm{C}-\mathrm{C}$ bond in the tail site and $1.43 \AA$ for $\mathrm{C}-\mathrm{C}$ bond in the thiophene site. The $\mathrm{C}-\mathrm{H}$ bond is $1.11 \AA$ and $\mathrm{C}-\mathrm{S}$ bond length is $1.67 \AA$.
As a first step of the formation of the conjugated P3HT backbone, two molecules are bonded each other from their thiophene sites. Our calculations showed that such a bonding leads to a $3.08 \mathrm{eV}$ molecule ${ }^{-1}$ energy gain in the two-molecule P3HT structure.

In the next step the formation of one-dimensional conjugated backbones of P3HT was investigated. For the 1D periodic P3HT case, shown in figure 3(B), calculated parameters $a=7.73 \AA$ and $b=19.93 \AA$ are in good agreement with previously reported values $[25,26]$ and with the results of XRD measurements of our experiments. Total energy calculations revealed that transition from two-molecular structure to one-dimensional conjugated backbone structure happens with a large energy gain in the structure (binding energy of $3.77 \mathrm{eV}$ molecule $\mathrm{e}^{-1}$ ). Therefore, it can be deduced that formation of one-dimensional conjugated backbone structure is highly favored over the other possible P3HT structures. In addition, electronically, one-dimensional conjugated backbone structure has a semiconducting ground state with a bandgap of $1.03 \mathrm{eV}$. To understand the formation of each P3HT layer the lateral interaction mechanism between one-dimensional conjugated backbones of P3HT was also investigated. Careful structural optimization calculations revealed that tail-tail interactions play an important role for the formation of a 2D periodic structure as given by STM measurements. Formation of two-dimensional sheet of P3HT occurs with an energy gain of $337 \mathrm{meV}$ per molecule. For the 2D P3HT structure, shown in figure 3(C), the lattice constant along the polymer repetition direction is $7.85 \AA$ which is in agreement with XRD measurements and the lattice constant along the alkyl side chain direction is $13.43 \AA$. It was also noticed that upon the formation of two-dimensional layers the energy band gap increases to $1.14 \mathrm{eV}$.

Next, how DCB and CHN molecules interact with P3HT layers was investigated. For this purpose, first the binding of the two molecules in vacuum was simulated. It is found that the DCB molecule has a planar structure in which $\mathrm{H}$ and $\mathrm{Cl}$ atoms are bonded to a benzene ring from $\mathrm{C}$ atoms. The $\mathrm{C}-\mathrm{C}$ 
A

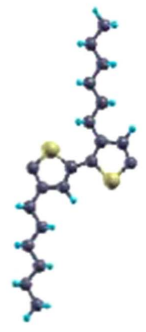

$\mathrm{B}$

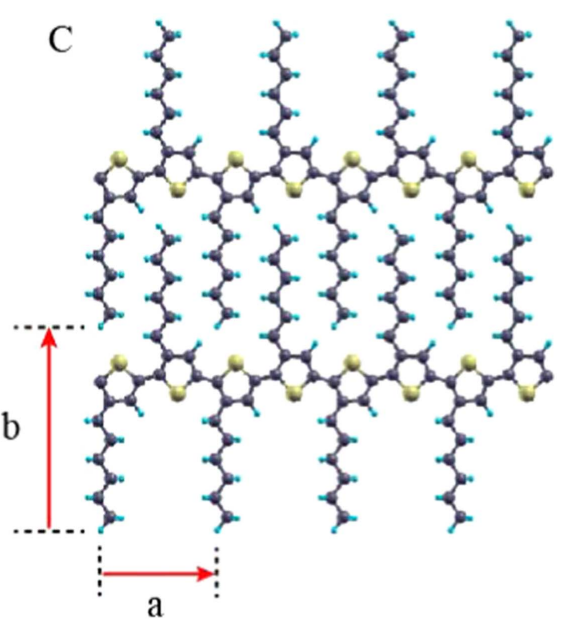

Figure 3. Geometry of P3HT molecules in (A) vacuum, (B) 1D periodic chain and (C) 2D periodic structure.

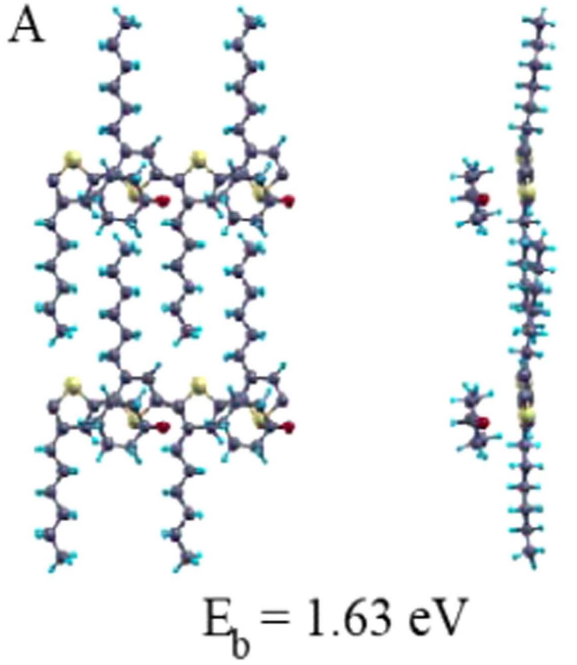

B

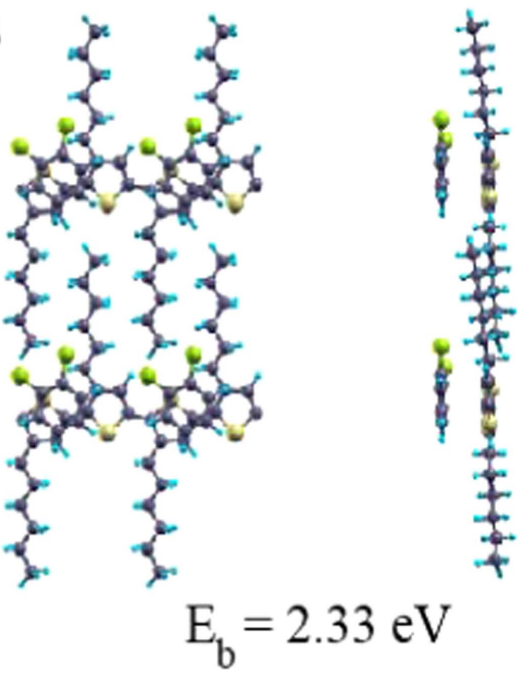

Figure 4. (A) CHN molecule on 2D P3HT layer. (B) DCB molecule on 2D P3HT layer.

bond length is $1.39 \AA$ and that of $\mathrm{C}-\mathrm{H}$ is $1.11 \AA$ while the $\mathrm{C}-\mathrm{Cl}$ bond length is $1.7 \AA$. The calculated HOMO-LUMO gap of DCB is $4.34 \mathrm{eV}$. Geometry optimization for $\mathrm{CHN}$ molecule showed that the $\mathrm{C}-\mathrm{C}$ bond length is $1.52 \AA$ while $\mathrm{C}-\mathrm{H}$ bond length is the same as in DCB molecule and the $\mathrm{C}-\mathrm{O}$ bond length is $1.21 \AA$. The HOMO-LUMO gap of the $\mathrm{CHN}$ molecule is $3.68 \mathrm{eV}$ corresponding to the wavelength of $336.9 \mathrm{~nm}$. As presented in figure 4 the binding energies of CHN and DCB molecules on a two-dimensional P3HT layer are $1.63 \mathrm{eV}$ and $2.33 \mathrm{eV}$ per unit cell, respectively. Therefore, it is obvious why DCB is a better solvent than CHN. However, our finding does not exclude the solving effect of $\mathrm{CHN}$ molecules at relatively low temperatures.

Next, possible stacking scenarios of two layers of P3HT were considered. As shown in figure 5 there are five different possible $\pi-\pi$ stacking orders for the P3HT layers. As presented in figure $5(\mathrm{C})$ energetically the most favorable one is $\mathrm{X}$-rotated stacking. However, other three stacking configurations shown in (B), (D) and (E), may also exist in the bulk form since their total energies are close to that of the $\mathrm{X}$-rotated stacking. It is worthwhile to note that the AA stacking (top-to-top) is the energetically least favorable configuration among the possible configurations. Therefore these findings explain why our pre-existing bulk P3HT structure is in a randomly ordered form.

Obviously, at the beginning of the experiment, the bulk structure of P3HT includes a mixture of these various stacking that is also seen in the STM measurements of our experiment. Interestingly, the stacking geometry of P3HT layers is strongly affected by the presence of solvent DCB molecules. As shown in figure 6 , the binding energies in Y-rotated and XY-rotated stacking are very close to each other and also for the shifted and X-rotated stacking the situation is the same. However, the AA stacking has the highest binding energy when DCB is located in between the P3HT layers. It can be concluded that when the solving process is completed, DCB molecules are mostly found at thiophene sites and they facilitate formation of AA stacking of P3HT layers. Moreover, as shown in figure 6, although DCB arranges the layers in AA stacking it also leads to a significant distortion in the tails of the P3HT layers. Therefore the presence of DCB molecules between P3HT layers is not 


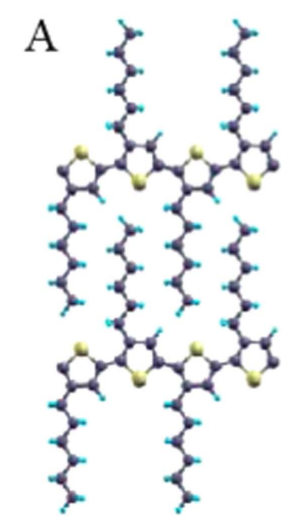

$\Delta \mathrm{E}=1.04 \mathrm{eV}$

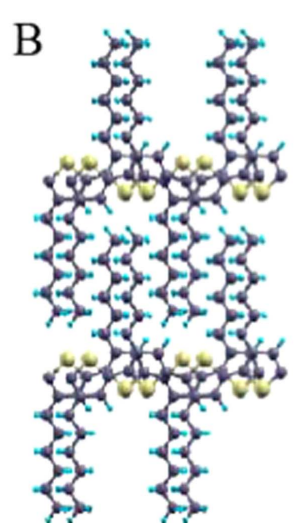

$\Delta \mathrm{E}=0.07 \mathrm{eV}$

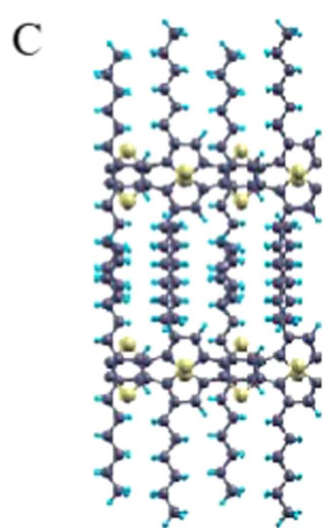

$\Delta \mathrm{E}=0 \mathrm{eV}$
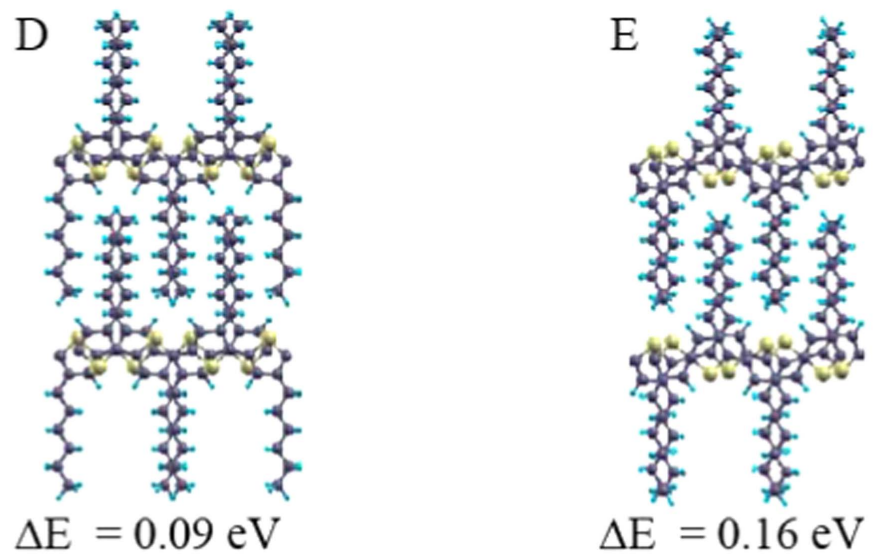

Figure 5. Bare bilayer stacking of 2D P3HT layers (A) AA, (B) Shifted, (C) X-rotated, (D) Y-rotated and (E) XY-rotated stacking.

A

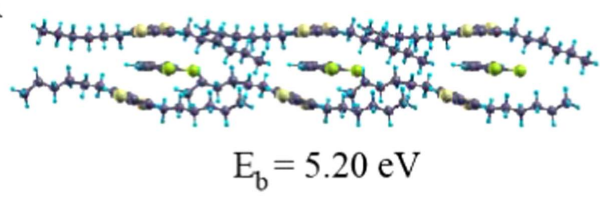

C

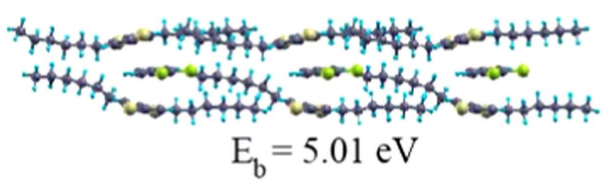

B

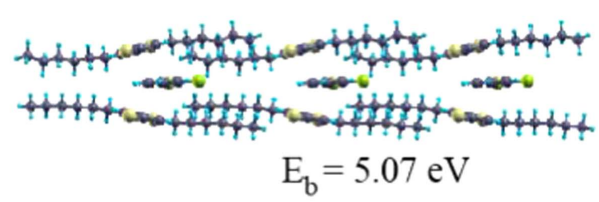

D

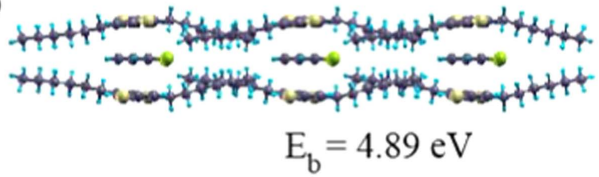

E

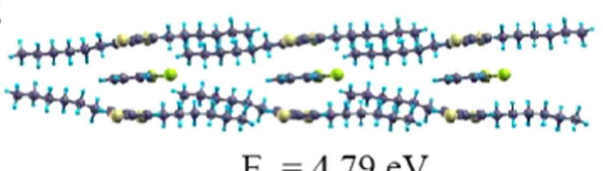

$\mathrm{E}_{\mathrm{b}}=4.79 \mathrm{eV}$

Figure 6. DCB molecule in between 2D P3HT layers in (A) AA, (B) Shifted, (C) X-rotated, (D) Y-rotated and (E) XY-rotated stacking.

enough for the formation of well ordered nucleus of nanowire. Then, understanding the role of $\mathrm{CHN}$ molecules in the DCB adsorbed P3HT is of importance. Figure 7(A) shows that in the vacuum state the most favorable geometry of DCB and $\mathrm{CHN}$ molecules is top-to-top configuration. However, in the presence of P3HT CHN molecules prefer to be adsorbed at tail sites with $1.86 \mathrm{eV}$ binding energy (see figure 7(B)). Our calculations also reveal that $\mathrm{CHN}$ molecules prefer to interact with DCB molecules from oxygen atom edge to hydrogen atom site of the molecules. It is seen that when $\mathrm{Cl}$ atoms interact with $\mathrm{O}$ atoms the binding energy is close to that of $\mathrm{O}_{-}$ $\mathrm{H}$ edge structure but due to the interaction of $\mathrm{O}$ atoms with the tail of P3HT layer there exists a distortion in P3HT layer. Therefore, apparently, CHN molecules behave as a glue at tails when it interacts with DCB. Therefore the coexistence of DCB and $\mathrm{CHN}$ leads to formation of three-dimensional lamellar stacking of the conjugated backbones (figure 8). This is in good agreement with our STM results. 
A

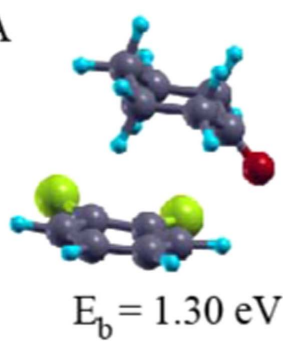

B

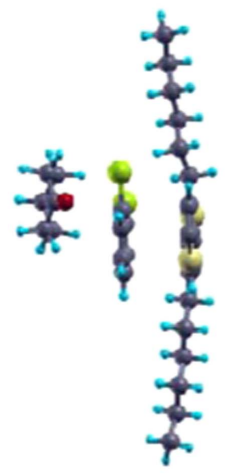

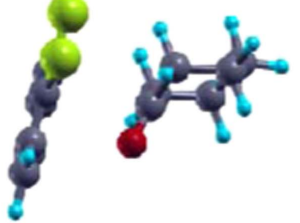

$\mathrm{E}_{\mathrm{b}}=1.16 \mathrm{eV}$

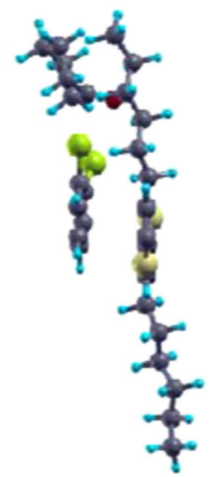

$\mathrm{E}_{\mathrm{b}}=1.81 \mathrm{eV}$

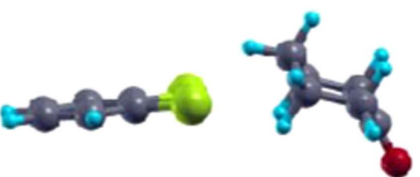

$\mathrm{E}_{\mathrm{b}}=0.78 \mathrm{eV}$

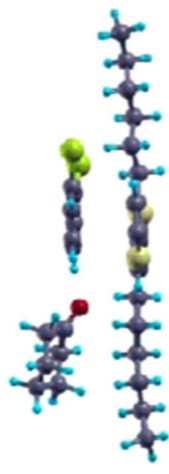

$\mathrm{E}_{\mathrm{b}}=1.86 \mathrm{eV}$

Figure 7. Interactions of $\mathrm{DCB}$ and $\mathrm{CHN}$ molecules in (A) vacuum in three different configurations, (B) P3HT single layer.

A

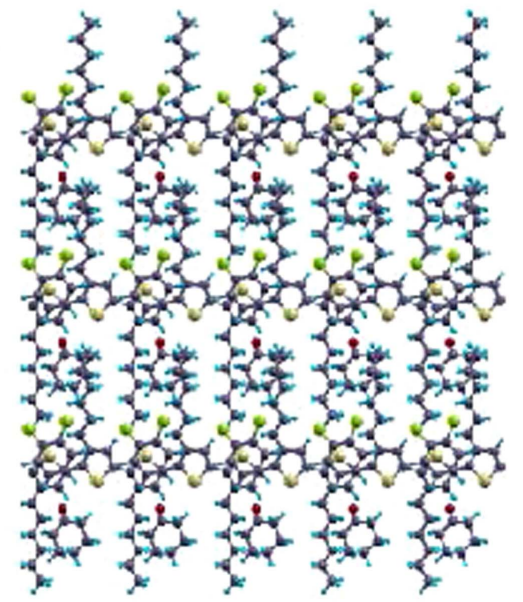

B

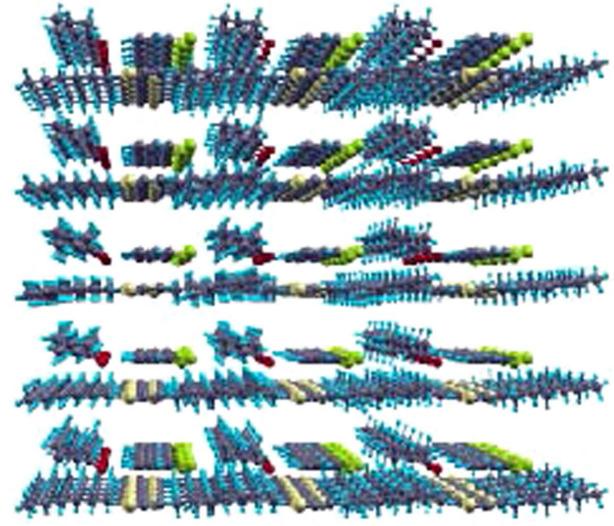

Figure 8. Bulk structure of P3HT. (A) Top and (B) side view of the bulk structure with DCB and CHN molecules. During the formation process of layered bulk P3HT, while thiophene sites are attached via DCB, tails are stabilized by CHN molecules. The inter-planar distance between two P3HT layers is $8.5 \AA$.

\subsection{Atomic force microscopy studies in P3HT nanowire evolution}

The growth mechanism of core-nanowire was investigated by AFM studies depending on incubation time upon mixture of CHN on the P3HT/DCB solution (figure 9). In order to comprehend the nanowire evolution, $\mathrm{P} 3 \mathrm{HT}$ (in DCB): $\mathrm{CHN}$ (10:7 ratio by volume) mixture was spin coated on mica substrate at regular intervals, from $1 \mathrm{~s}$ to $30 \mathrm{~min}$ The color of the supernatant was changed from brown to dark purple by diffusion of CHN toward the bottom of the vial upon adding CHN on the P3HT/DCB solution.

In the first second of the mixture, the sample was taken from interface of CHN and bulk P3HT. The AFM image in figure 9(A) demonstrates the initiated nanowires. The length and diameter of nanowires were measured as more than $10 \mu \mathrm{m}$ and around $40 \pm 5 \mathrm{~nm}$, respectively. Furthermore, nanowires form a network like surface with $1,1 \mathrm{~nm}$ RMS roughness. Figures 9(B)-(G) illustrates the growth morphological changes of the wires with various incubation times. According to the figure 9(B), net like slim nanowires with the thickness of $35 \pm 9 \mathrm{~nm}$ are distinguished slightly after the first minute. In addition, there are many pits with diameter between 0.2 and $1 \mu \mathrm{m}$ in AFM images. The sizes of these pits depend on growth mechanism of nanowires and the film formation in the P3HT solution. In all AFM images the pits contain few nanowires as well. Kramer et al [16] suggest that 

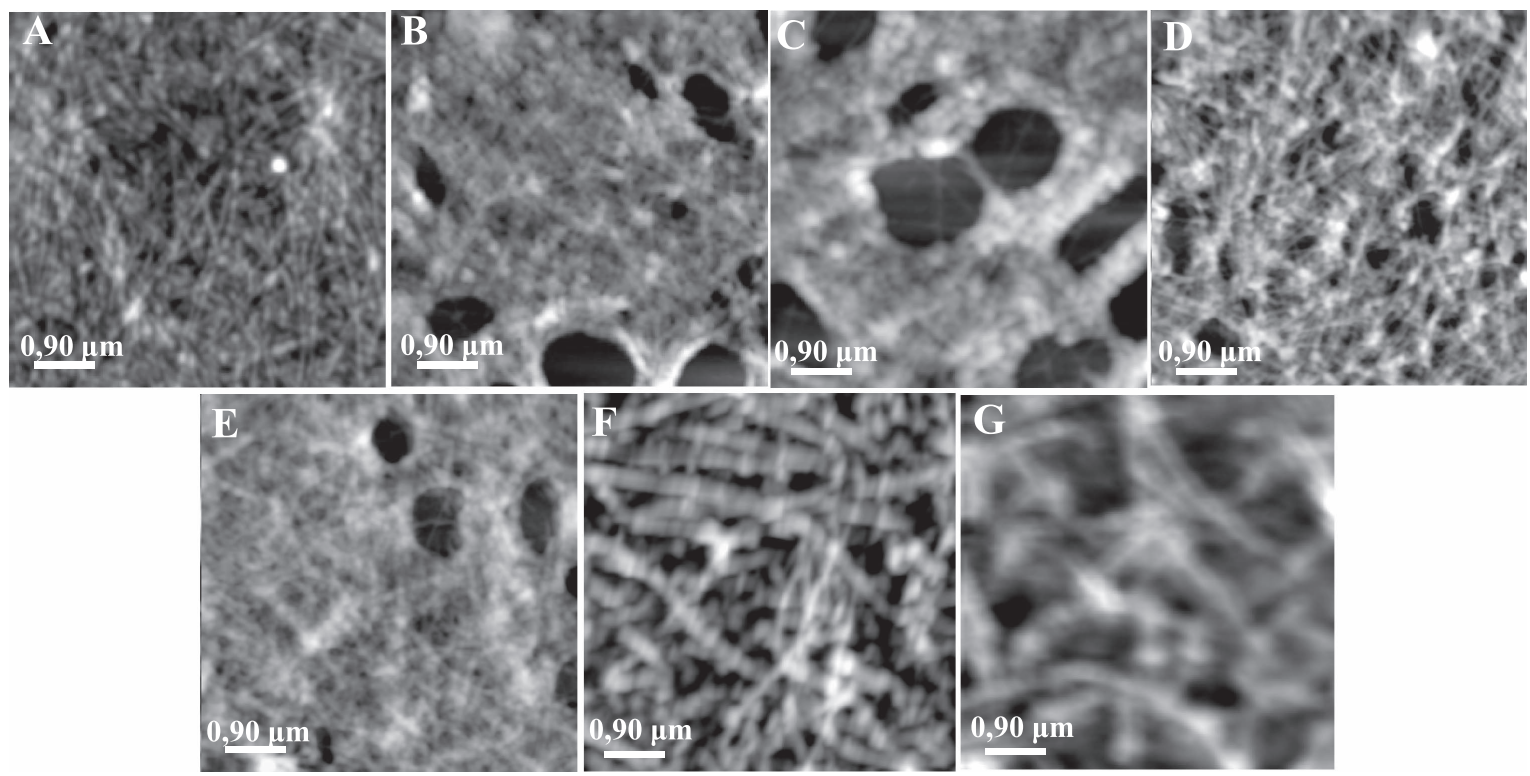

Figure 9. AFM images of nanowires formed at different incubation time after the addition of CHN (10:7 in volume ratio) on P3HT/DCB solution. (A) $1 \mathrm{~s} \mathrm{~B}) 1 \mathrm{~min}$, (C) $2 \mathrm{~min}$, (D) $4 \mathrm{~min}$, (E) $8 \mathrm{~min}$, (F) $15 \mathrm{~min}$ and (G) $30 \mathrm{~min}$.

Table 1. Surface properties of P3HT-nanowire film topographies in different time interval.

\begin{tabular}{lccc}
\hline & $S_{\mathrm{a}}(\mathrm{nm})$ & $S_{\mathrm{q}}(\mathrm{nm})$ & $S_{\mathrm{z}}(\mathrm{nm})$ \\
\hline $\mathbf{1 ~ s}$ & 1.1 & 1.5 & 15.6 \\
$\mathbf{1}$ min & 6.6 & 8.7 & 64.5 \\
$\mathbf{2} \mathbf{~ m i n}$ & 9.8 & 12 & 70 \\
$\mathbf{4} \mathbf{~ m i n}$ & 10.3 & 13 & 93.8 \\
$\mathbf{8} \mathbf{~ m i n}$ & 11.5 & 15 & 121 \\
$\mathbf{1 5} \mathbf{~ m i n}$ & 6.1 & 7.5 & 48 \\
$\mathbf{3 0}$ min & 1.2 & 1.5 & 12 \\
\hline
\end{tabular}

these pits are created by the CHN included in the P3HT solution, and they migrate from the surface during the spin coating.

As seen from the AFM images the number of nanowires increase significantly with increasing the interval time in growth solution. The thickness of nanowires is between in the range of $26-100 \mathrm{~nm}$ at the end of the second minute (figure $9(\mathrm{C})$ ) and then reaches to $150 \pm 5 \mathrm{~nm}$ at the fourth minute (figure 9(D)). This increment in the thickness is due to formation of the nanowires as a bundle. The sizes of the nanowire at the end of $8 \mathrm{~min}$ are not significantly change (AFM image in figure 9(E)). As mentioned above the size and depth of these pits mostly depend on time interval of nanowire growth. The pits appear in the few minutes of growth and then shrink or completely disappear from surfaces when the time reach to 15 and $30 \mathrm{~min}$ (figures $9(\mathrm{~F})$ and $(\mathrm{G})$ ). Those results indicate that the $\mathrm{CHN}$ is exhausted during the nanowire formation. Decreasing the amount of CHN means to increase of the number of nanowires.

Table 1 shows roughness values of the nanowire film surfaces after different interval times. They were measured in terms of average roughness $\left(S_{\mathrm{a}}\right)$, root mean square roughness
$\left(S_{\mathrm{q}}\right)$ and ten point height $\left(S_{\mathrm{z}}\right)$. Average height was determined as the average of ten different points.

The average roughness $\left(S_{\mathrm{a}}\right)$ of the nanowire surface in the first second is $1.1 \mathrm{~nm}$. From 1 to $8 \mathrm{~min}$, the roughness significantly increases to $7.6 \mathrm{~nm}(1 \mathrm{~min}), 9.8 \mathrm{~nm}(2 \mathrm{~min})$, $10.3 \mathrm{~nm}(4 \mathrm{~min})$ and $11.5 \mathrm{~nm}(8 \mathrm{~min})$. On the other hand, at the higher interval time the roughness decreases to $5.5 \mathrm{~nm}$ at 15 th minute and $1.2 \mathrm{~nm}$ at $30 \mathrm{th}$ minute. It can be concluded that higher interval time can lead to form better nanowire film surface.

From all these results we can emphasis that the nanowire film formation in the solution continues to the fourth minute, and then stops slowly due to the lack of the free P3HT molecules that contribute to the nanowire formation. The main difference of the wires was the change of thickness with time. The underlying reason for this result can be described as core P3HT nanowires were formed at the $\mathrm{CHN}$ interface upon P3HT molecules meet with CHN. The free P3HT molecules around the nanowires join to the main chains. After the eighth minute P3HT nanowires begin to form bundles by van der Waals forces due to the $\pi$-electron system.

The formation of nanowires was also investigated in detailed using a high resolution STM. An STM image of nanowires were shown figure $10(\mathrm{~A})$ as a bundle on a highly ordered pyrolytic graphite (HOPG) substrate. Within the region of the HOPG surface, nanowires are aligned in structures that extend well over $100 \mathrm{~nm}$. From the image these nanowires constitute a bundle with diameter of $55 \mathrm{~nm}$. This is in good agreement with AFM images (figure 9). The enlarged area of figure 10(A) is given in figure 10(B). Figure 10(C) shows a packing arrangement consistent with the experimental data supported by DFT calculations. We suggest that the brighter spots in the STM image correspond to the thiophene units, while the fainter regions observed on the either side of the brighter areas are attributed to the alkyl chains. 

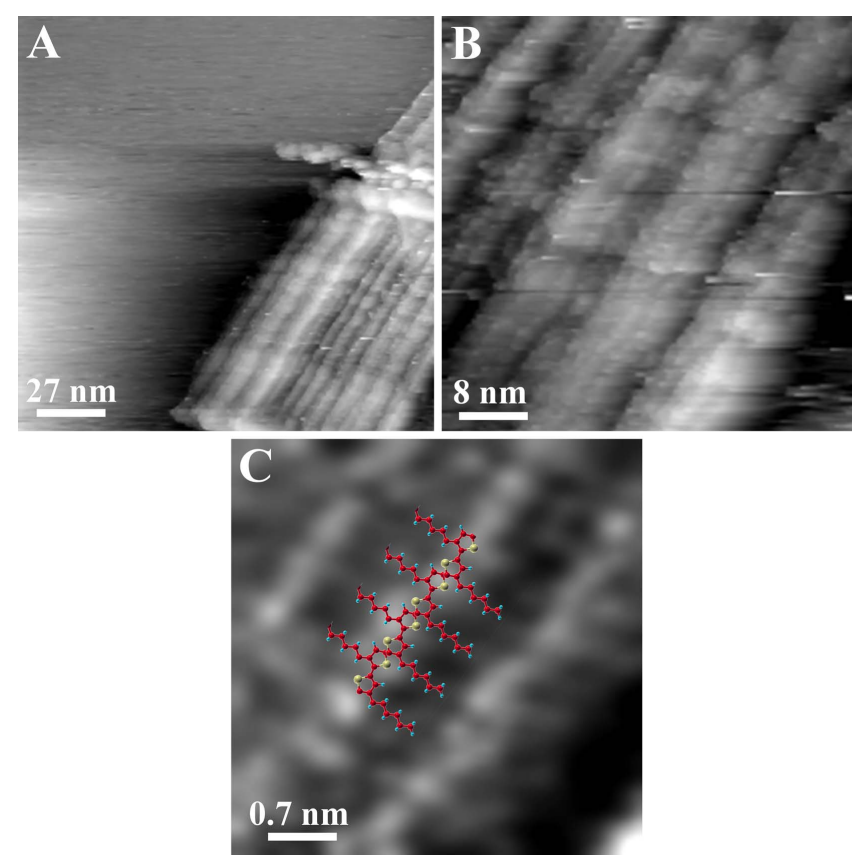

Figure 10. STM images of ordered assemblies of nanowires. (A) Large area image, (B) higher magnification of (A) and (C) magnified STM image overlaid with packing of four P3HT chains.
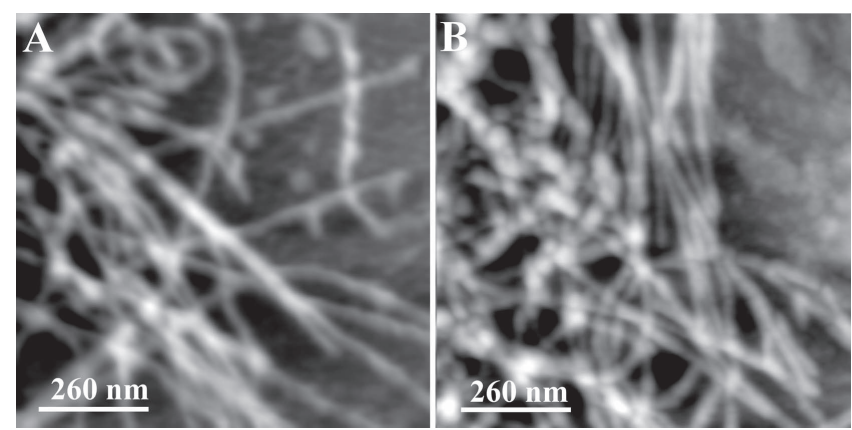

Figure 11. AFM images of P3HT nanowires formed in vials with (A) $15 \mathrm{~mm}$ diameter and (B) $11 \mathrm{~mm}$ diameter respectively.

According to the images, the diameter and the height of nanowires defined by the chain interaction between P3HT polymers and the $\pi-\pi$ stacking of polymers respectively. Depending on the AFM images, the height of wires changes between 2 and $4 \mathrm{~nm}$ indicating 4-8 polymer overlapping.

Consequently, STM investigations clarify that length of the nanowires were along the polymer backbone and determined by the number of interacted polymer backbones by $\pi-$ $\pi$ stacking at the edges. (figures $2(\mathrm{~A})$ and $10(\mathrm{C})$ ).

\subsection{Surface area dependence of molecular self-assembly}

The interaction area of P3HT/CHN interface was changed in order to understand the nanowire formation mechanism in detail. The first sample had a $0.95 \mathrm{~cm}^{2}$ interaction area between P3HT:DCB and CHN whereas the second sample had an interface with $1.77 \mathrm{~cm}^{2}$. Prepared solutions were left during the night in order to let the all P3HT molecules to form nanowires and participate. Consequently, the nanowire containing solutions were spin coated on mica substrate. Figure 11 demonstrates the AFM images of the nanowires formed at the two different interface areas. The diameter of P3HT-wires were measured as $23 \pm 5 \mathrm{~nm}$. Subsequently, from the AFM and STM images it was observed that bundles were formed by combination of two or more wires. Unlike the larger one, there is a wide range of diameters of individual nanowires from 30 to $70 \mathrm{~nm}$ at the sample with small interface area. Since diffusion of CHN is slower than the wire creation, the core-wires are formed at the $\mathrm{CHN} / \mathrm{P} 3 \mathrm{HT}$ interface, instantly. Both the core wires and CHN diffuse through the bulk P3HT with the time. A smaller interaction area of CHN/P3HT means less formation of core-wire. These results are indication of the contribution of free $\mathrm{P} 3 \mathrm{HT}$ polymers to the thickness of wires. Therefore, thicker nanowires were obtained in the vial with the smaller diameter. As a result, wire thickness is controlled by interface area, in other words by nanowire creation velocity.

\section{Conclusions}

Formation mechanism and evaluation of P3HT nanowires by self-assembly $\pi-\pi$ stacking and chain to chain interaction induced by poor solvent were investigated. Morphological, spectroscopic and structural properties were studied by using AFM STM, UV-vis absorption spectroscopy and XRD measurements. Experimental results were correlated with computational DFT calculations. The STM results suggested that the length of the nanowires were determined by polymer repetition degree (polymer chain length) and partially $\pi-\pi$ stacking at the ends of polymers. Width and height of the wires were determined by chain-chain interactions and stacking of several layers of polymers by $\pi-\pi$ interactions perpendicular to the substrate respectively. Polymer chain repetition was determined to be dominant on polymer nanowire elongation; however, STM studies confirm that $\pi-\pi$ stacking on the polymer edges was also effective on the growth. It was also determined that wire formation occurred at the $\mathrm{DCB} / \mathrm{CHN}$ interface and area of the interface is strongly effective on the rate of the wire formation. Our calculations showed that well ordered top-to-top stacking of the thiophene rings is provided by DCB molecules. However, the tail groups of the polymer lead to distortion due to the thermal motion at high temperatures. In addition the tail-tail interactions that avoid nucleation of the P3HT core lamellar structure are stabilized by $\mathrm{CHN}$ poor solvent molecules. These results revealed that solvophobic interactions are strongly effective on the self-assembly of the molecules. Consequently, our spectroscopy and microscopy studies supported by theoretical calculations reveal that, in the presence of both poor and good solvents, in the self-assembled P3HT nanowire network, the nanowire axis is elongated through the polymer chain direction. Additionally, STM and XRD data support that $\pi-\pi$ stacking is also effective in the wire elongation at the polymer chain ends. 


\section{Acknowledgments}

This work was supported by the Flemish Science Foundation (FWO-Vl) and the Methusalem foundation of the Flemish government. Computational resources were provided by TUBITAK ULAKBIM, the High Performance and Grid Computing Center (TR-Grid e-Infrastructure), and the HPC infrastructure of the University of Antwerp (CalcUA), a division of the Flemish Supercomputer Center (VSC), which is funded by the Hercules Foundation. HS is supported by a FWO Pegasus-Long Marie Curie Fellowship. HS and RTS acknowledge support from TUBITAK through Project No. 114F397. Also, DA is supported by the Scientific Research Project Fund of Ege University (Project Nr: 12GEE011).

\section{References}

[1] Hoppe H and Sariciftci N S 2011 Organic solar cells: an overview J. Mater. Res. 19 1924-45

[2] Friend R H et al 1999 Electroluminescence in conjugated polymers Nature 397 121-8

[3] Zhang C, Yan Y, Sheng Zhao Y and Yao J 2013 Synthesis and applications of organic nanorods, nanowires and nanotubes Annu. Rep. C 109211

[4] Kamkar D A, Wang M and Fred Wudl T-Q N 2012 Single nanowire OPV properties of a photoconductive AFM ACS Nano 6 1149-57

[5] Wu P-T, Xin H, Kim F S, Ren G and Jenekhe S A 2009 Regioregular poly(3-pentylthiophene): synthesis, selfassembly of nanowires, high mobility field effect transistors, and efficient photovoltaic cells Macromolecules 42 8817-26

[6] Im J-H et al 2010 Bulk heterojunction solar cells based on preformed polythiophene nanowires via solubility-induced crystallization J. Mater. Chem. 207398

[7] Chen S and Ni J 1992 Structure/properties of conjugated conductive polymers: I. Neutral poly (3-alkythiophene)s Macromolecules 25 6081-9

[8] Johnson C E and Boucher D S 2014 Poly(3-hexylthiophene) aggregate formation in binary solvent mixtures: an excitonic coupling analysis J. Polym. Sci. B 52 526-38

[9] Kiriy N et al 2003 One-dimensional aggregation of regioregular polyalkylthiophenes Nano Lett. 3 707-12

[10] Dierckx W, Oosterbaan W D, Bolsée J-C, Maes W, Vanderzande D and Manca J 2014 Poly(3-alkylthiophene) nano-fibers for optoelectronic devices J. Mater. Chem. C 25730
[11] Surin M et al 2006 Relationship betweenthe microscopic morphology and the charge transport properties in poly(Shexylthiophene) field-effect transistors J. Appl. Phys. 100 $1-6$

[12] Schwarz K N, Kee T W and Huang D M 2013 Coarse-grained simulations of the solution-phase self-assembly of poly(3hexylthiophene) nanostructures Nanoscale 52017

[13] Brown P et al 2003 Effect of interchain interactions on the absorption and emission of poly (3-hexylthiophene) Phys. Rev. B 67 1-16

[14] Rughooputh S and Hotta S 1987 Chromism of soluble polythienylenes J. Polym. 25 1071-8

[15] Ye Z, Yang X, Cui H and Qiu F 2014 Nanowires with unusual packing of poly(3-hexylthiophene)s induced by electric fields J. Mater. Chem. C 26773

[16] Kramer T J, Chew A R, Schiros T, Kymissis I and Herman I P 2013 Poly-(3)hexylthiophene nanowire networks for versatile fabrication of bulk heterojunctions with increased active volume J. Vac. Sci. Technol. B 31 031209

[17] Wirix M J M, Bomans P H H, Friedrich H, Sommerdijk N A J M and de With G 2014 Threedimensional structure of P3HT assemblies in organic solvents revealed by cryo-TEM Nano Lett. 14 2033-8

[18] Ordejon P, Artacho E and Soler J M 1996 Self consistent order-N density-functional calculations for very large systems Phys. Rev. B $\mathbf{5 3} 4$

[19] Soler M et al 2002 The SIESTA method for ab initio order-N materials J. Phys. Condens. Matter 14 2745-79

[20] Kohn W and Sham L J 1965 Self-consistent equations including exchange and correlation effects Phys. Rev. 140 $1133-8$

[21] Perdew J P and Zunger A 1981 Self-interaction correction to density-functional approximations for many-electron systems Phys. Rev. B 23 5048-79

[22] Perdew J P and Wang Y 1992 Accurate and simple analytic representation of the electron-gas correlation energy Phys. Rev. B 45 13244-9

[23] Ceperley D M and Alder B J 1980 Ground state of the electron gas by a Stochastic method Phys. Rev. Lett. 45 $566-9$

[24] Troullier N and Martins J L 1991 Efficient pseudopotentials for plane wave calculations: II. Operators for fast iterative diagonalization Phys. Rev. B 43 8861-9

[25] Melis C, Mattoni A and Colombo L 2010 Self assembling of poly(3-Hexylthiophene) J. Phys. Chem. C 115 $576-81$

[26] Colle R, Grosso G, Ronzani A and Zicovich-Wilson C M 2011 Structure and $\mathrm{x}$-ray spectrum of crystalline poly(3hexylthiophene) from DFT-van der Waals calculations Phys. Status Solidi Basic Res. 248 1360-8 